\title{
Essais
}

ESSAIS

Revue interdisciplinaire d'Humanités

$11 \mid 2017$

Fictions de l'identité

\section{La Cité de Dieu de Saint Augustin : une histoire identitaire en réponse à un trouble politique?}

\section{Philippe Cournault}

\section{(2) OpenEdition}

\section{Journals}

Édition électronique

URL : http://journals.openedition.org/essais/3246

DOI : $10.4000 /$ essais.3246

ISSN : 2276-0970

Éditeur

École doctorale Montaigne Humanités

\section{Édition imprimée}

Date de publication : 15 mai 2017

Pagination : 17-28

ISBN : 979-10-97024-01-7

ISSN : 2417-4211

\section{Référence électronique}

Philippe Cournault, «La Cité de Dieu de Saint Augustin : une histoire identitaire en réponse à un trouble politique? », Essais [En ligne], 11 | 2017, mis en ligne le 14 octobre 2020, consulté le 21 octobre 2020. URL : http://journals.openedition.org/essais/3246 ; DOI : https://doi.org/10.4000/essais.3246 


\section{La Cité de Dieu de Saint Augustin : Une histoire identitaire en réponse à un trouble politique?}

\section{Philippe Cournault}

D'aucuns prétendent que les grandes œuvres sont celles qui échappent à la classification en genres. Et il faut reconnaître que les deux ouvres les plus justement célèbres d'Augustin - Les Confessions et La Cité de Dieu - ne se laissent pas enfermer comme les nombreuses autres œuvres ${ }^{1} \mathrm{du}$ prolixe docteur dans les genres codifiés du traité théologique, du commentaire exégétique, du sermon ou de la lettre. Ainsi, malgré une mode récente mais tenace qui attribue à Augustin l'invention du genre autobiographique avec les Confessions, même les neuf premiers livres de cette œuvre où il suit le fil de son enfance et de son adolescence $^{2}$ recèlent une variété de genres littéraires et, surtout, un propos et une structure profonde qui dépassent ceux de l'autobiographie ${ }^{3}$. De son côté, La Cité de Dieu semble hésiter entre les genres de l'apologétique et de la narratio catéchétique et les commentateurs ont mis l'accent tantôt sur l'un tantôt sur l'autre de ces genres.

Plus largement, les grandes œuvres ne se laissent pas facilement enfermer dans des formules séduisantes qui pourraient sembler capables de les définir. Si donc il est tentant de définir La Cité de Dieu comme une histoire identitaire en réponse à un trouble politique, il convient d'être prudent avec cette formule. Ce qui ne signifie pas qu'on s'en démarquera a priori : au contraire, on pourra, d'abord, expliciter en quoi cette formule peut s'appliquer à La Cité de Dieu mais, se souvenant que l'on a affaire à une grande œuvre, on veillera à ne pas l'enfermer dans cette formule et on gardera à l'esprit d'examiner en quoi La Cité de Dieu ne se réduit pas à ces termes.

1 Pour une liste à peu près exhaustive des ouvres d'Augustin, voir le tableau récapitulatif de H.-I. Marrou dans Augustin et l'augustinisme (Paris, Seuil, 1968) pourtant à réactualiser avec les découvertes récentes de François Dolbeau.

2 Au sens latin de cet âge de la vie (adulescentia) soit entre 17 et 30 ans.

3 Voir l'introduction, la traduction et les notes de P. Cambronne dans son édition des Confessions dans la Bibliothèque de la Pléiade (Paris, Gallimard, 1998). 
Sans doute convient-il, en préalable, de rappeler le plan de La Cité de Dieu : l'œuvre présente, en effet, ce paradoxe qu' elle est à la fois très connue, très citée et, en fait, assez peu lue dans le détail et encore moins dans son intégralité. Augustin annonce et souligne, à de nombreuses reprises, ce plan. Dans Les Révisions ${ }^{4}$, il marque clairement une division en deux parties des vingt-deux livres, les dix premiers étant consacrés à une réfutation des Païens et les douze derniers à un exposé des vues chrétiennes. En fait, on peut légitimement mettre à part le premier livre consacré à l'événement qui a déclenché la rédaction de l'œuvre - le Sac de Rome par les Goths en 410 - et à la critique qui s'en est suivie des Chrétiens par les Païens. Ces critiques sont, en effet, réfutées méthodiquement dans les livres II à X. Cette réfutation aboutissant, selon la logique même de l'apologétique, à l'affirmation de la supériorité de la foi chrétienne et de ses Écritures, les livres XI à XXII de la seconde partie parcourent non seulement l'histoire universelle de l'humanité mais aussi la proto-histoire angélique pour dégager, à l'œuvre dans cette Histoire et selon les mots mêmes d'Augustin, "les origines, le développement et les fins $s^{5}$ " de deux cités antagonistes : la Cité de Dieu et la Cité terrestre. Augustin tient visiblement à l'équilibre de ces trois moments de l'histoire universelle ${ }^{6}$ puisque, sur les douze livres de la seconde partie, il consacre quatre livres à l'origine de ces deux cités dans le monde angélique et dans la naissance de l'être humain (ce sont les livres XI à XIV), quatre autres livres au développement de ces deux cités dans l'histoire humaine (ce sont les livres XV à XVIII) et les quatre derniers livres aux fins des deux cités révélées par le Jugement Dernier et s'ouvrant sur l'éternité (ce sont les livres XIX à XXII).

Ce plan très général en tête, on peut d'abord vérifier en quoi La Cité de Dieu est un essai d'histoire identitaire en réponse à un trouble politique et, pour ce faire, en entreprendre un rapide parcours selon le plan qui vient d'être rappelé.

Le livre I que nous avons mis à part tout à l'heure ancre tout le développement des vingt et un livres suivants dans l'événement du Sac de Rome par le Goth Alaric le 24 août 410. Or cet événement, s'il n’a pas été un véritable trouble politique, a été perçu comme tel par les Païens comme par les Chrétiens et, en lui consacrant tout le livre inaugural de La Cité de Dieu, Augustin fait non seulement des livres II à X mais aussi des livres XI à XXII comme une réponse à ce trouble politique.

4 Voir Saint Augustin, Révisions, II, XLIII, 1.

5 Saint Augustin, La Cité de Dieu, X, XXXII, 4 ; XI, I, XVIII, I... : " exortus et procursus [excursus] et debiti fines » cité, comme dans la suite de l'article, selon l'édition de la Bibliothèque Augustinienne (Euvres de saint Augustin, cinquième série, n 33-37, Desclée de Brouwer, 1959-1960).

6 Voir G. P. O’Daly, Augustine's City of God. A Reader's guide, Oxford University Press I Clarendon Press, «5. La structure de l'œuvre », Oxford, 2004. 
Selon les historiens contemporains de l'événement, l'interprétation diverge beaucoup sur ce qui s'est passé ce 24 août 410 à Rome et sur son importance. Certains, en effet, parlent de trois jours de mise à sac et de pillage alors que d'autres minimisent ; ainsi ne trouverait-on que quelques traces d'incendie et l'historien Orose en fait un non-événement : "nihil factum !? ". Globalement, comme on le verra tout à l'heure, les Païens imputant aux Chrétiens la responsabilité de la chute de la Ville éternelle ont tendance à noircir la catastrophe tandis que les Chrétiens ont tendance à la minimiser. André Piganiol qui a consacré tout un ouvrage à l'événement du Sac de Rome commence par rappeler que Rome n'est plus, depuis longtemps, capitale d'Empire, que les empereurs n'y séjournent plus, que le Sénat n'y demeure plus que comme une cour d'enregistrement, que la population, avant même le 24 août 410 , y a beaucoup diminué ${ }^{8}$. Et l'on retrouve cette même tendance à relativiser l'ampleur du drame dans la présentation plus récente qu'en fait Serge Lancel dans sa récente biographie d'Augustin'. Il reste que l'événement est vécu comme un traumatisme et le cri de saint Jérôme en plein commentaire d'Ézéchiel depuis Bethléem transcrit des cris d'apocalypse partagés par beaucoup d'habitants de Rome en fuite vers l'Afrique, vers l'Orient et jusqu'à Bethléem précisément :

Elle est conquise, la Ville qui a conquis l'Univers ! ${ }^{10}$

Horreur! Horreur ! L'univers s'écroule !11

Et c'est bien un trouble politique au sens étymologique du terme qui s'empare des habitants puisque la cité ne tenait debout que selon un contrat d'échange réciproque au terme duquel les Dieux n'avaient accordé à la Ville un accroissement territorial et ne lui accordaient encore une protection matérielle qu'en échange de la perpétuation des cultes. Et l'Empire devenu chrétien, en ordonnant progressivement, surtout sous Théodose, l'interdiction des cultes païens jusqu'aux jeux et au théâtre, avait trouvé bon de christianiser le contrat, parlant d'une protection du Dieu unique sur la Ville éternelle dans les mêmes termes que les Païens ${ }^{12}$. C'est à tel point que, tandis que, dans une certaine tradition, Rome incarnait encore, après Babylone, la puissance du mal, pour la plupart des Romains chrétiens, selon une autre tradition apocalyptique remontant au livre de Daniel, la chute de Rome, quatrième empire, inaugurerait la fin du monde ! Catastrophe pour les Païens, le Sac de Rome du 24 août 410 ne l'est donc pas moins pour un certain nombre de Chrétiens, la

7 Orose, Histoires, VII, LX, 1.

8 Voir André Piganiol, Le Sac de Rome, Paris, Albin Michel, 1964¹, 1972², p. 60-70.

9 Voir Serge Lancel, Saint Augustin, Fayard, 1999.

10 Jérôme, Epistula ad Principiam uirginem (= Ep. CXXVII), 12 : "Capitur Vrbs que totum cepit orbem».

11 Jérôme, Epistula, CXXVIII, 4 : «Proh nefas, orbis terrarum ruit».

12 Voir F. Paschoud, Roma aeterna : études sur le patriotisme romain dans l'Occident latin à l'époque des grandes invasions, Institut Suisse de Rome, 1967. 
résistance de l'Empire Romain et, d'une façon emblématique, de Rome aux invasions barbares étant devenue le signe de la faveur du Dieu unique, de sa Providence, de sa puissance et, pour tout dire, de l'autorité du christianisme. C'est donc dans un état de doute profond que nombre de Romains chrétiens se sont réfugiés sur les côtes de l'Afrique, de même que nombre d'aristocrates païens cultivés, profitant de la fragilité des Chrétiens pour les faire douter : en interdisant les cultes païens qui garantissaient, par contrat divin, la protection de la Cité, les Chrétiens auraient provoqué la chute matérielle de Rome. André Mandouze, supposant la fragilité toute particulière des nouveaux convertis va jusqu'à penser que "des nouveaux convertis retournaient aux temples demander aux dieux de leurs ancêtres ce que le dieu unique ne leur avait pas accordé ${ }^{13}$ ». C'est pour conforter ces demi-chrétiens hésitants réfugiés dans son diocèse et aussi pour tenter de convertir définitivement quelques Païens tentés par le christianisme qu'Augustin écrit $L a$ Cité de Dieu et réfute dans les premiers livres la sévère critique païenne : avec l'interdiction des cultes païens, les Chrétiens seraient responsables du Sac de Rome compris comme le trouble politique suprême puisque c'est la Cité-monde qui serait tombée.

À part, le livre I, loin d'être secondaire, est au fondement même de tout l'édifice de La Cité de Dieu, puisqu'il se situe au cour même du trouble politique qu'a été, pour ceux qui l'ont vécu, le Sac de Rome du 24 août 410 et que les vingt et un livres suivants n'ont pour seul objectif que de répondre à ce trouble, plus ou moins explicitement. Ces vingt et un livres suivants semblent, quant à eux, proposer, en réponse au trouble politique du Sac de Rome, une construction identitaire : la construction d'une Auctoritas chrétienne. Cette construction s'opère sur la déconstruction préalable de l'auctoritas païenne aux livres II à IX, culmine au livre $\mathrm{X}$ avec la démonstration de l'Auctoritas chrétienne et est mise en œuvre tout au long des livres XI à XXII de la seconde partie, puisque c'est grâce à l'Auctoritas des Écritures chrétiennes que l'histoire universelle y est parcourue et qu'elle semble s'acheminer vers le triomphe de la seule Cité de Dieu. Reprenons dans l'ordre ce qui pourrait donc passer pour une construction identitaire.

Les livres II à IX sont une opération de déconstruction de l'auctoritas païenne jusque dans ce qui fait l'identité même du paganisme aux yeux d'Augustin à savoir le paganisme religieux et plus précisément le culte polythéiste ainsi que la philosophie et la culture seulement dans la mesure où elles justifient ce paganisme religieux. En effet, les livres II, III et IV restent encore très proches de l'accusation païenne et y répondent en montrant que les dieux du paganisme n'ont jamais vraiment protégé Rome ni du mal moral (c'est le livre II), ni du mal matériel (c'est le livre III) pas plus qu'ils n'ont pu assurer son extension

13 André Mandouze, Saint Augustin : L'Aventure de la Raison et de la Grâce, "Chapitre VI. Les Métamorphoses de Rome ", Paris, Études Augustiniennes, 1968. 
spatiale (c'est le livre IV) ou temporelle (c'est le livre V) ${ }^{14}$. Mais, prolongeant la critique du polythéisme entamée dans ce livre V, les livres VI et VII continuent l'entreprise de démolition du paganisme jusque dans son identité même. Et comme si des Païens cultivés tentés par le christianisme prétendaient sauver de cette déconstruction au moins le sommet de l'édifice païen - le platonisme - qui, par sa démonologie, véritable théologie des intermédiaires, servait de caution philosophique au culte polythéiste, Augustin s'attaque dans les livres VIII, IX et X à ce sommet et en tire même l'occasion de démontrer, sur ce point central de la médiation - donc sur tout l'ensemble -, la supériorité de l'Auctoritas du christianisme. En effet, après avoir reconnu l'éminente valeur des Platoniciens au livre VIII, Augustin déconstruit au livre IX la médiation des démons selon Apulée pour y substituer la seule véritable médiation du Christ. C'est là le pivot de l'œuvre ${ }^{15} \mathrm{car}$ il peut en déduire, au livre X, la fausseté des théurgies et des sacrifices du système de Porphyre pour leur préférer les miracles des anges en judaïsme et en christianisme et le seul sacrifice du Christ. En un même mouvement, profitant des prétendues impasses et lacunes de l'auctoritas du paganisme, Augustin a donc démontré l'Auctoritas du christianisme, passant insensiblement de la déconstruction de l'identité païenne à la construction identitaire chrétienne. Ce faisant, il s'est situé au cœur même de l'apologétique tant dans sa démarche que dans son objectif : démontrer la supériorité du christianisme sur le paganisme. Jean-Claude Guy, parlant dans Unité et Structure logique de La Cité de Dieu, d'une "phénoménologie de l'incroyance en marche vers la foi ${ }^{16}$ ", avait, en son temps, perçu, à la fois, la solide structure de l'œuvre et son incroyable jeu de subtiles transitions.

La rupture est plus nette avec les livres XI à XXII de la seconde partie de La Cité de Dieu puisqu'Augustin commence, au livre XI, un immense parcours de l'histoire universelle. Mais en même temps, la continuité est tout aussi nette avec le livre X, livre pivot, puisque ce parcours se fait sous le regard exclusif de La Bible, des Écritures chrétiennes considérées désormais comme la seule Auctoritas, comme l'a démontré le livre X, et alors que les auteurs païens étaient la seule référence des livres I à X. La référence aux seules Écritures chrétiennes considérées comme la seule Auctoritas est donc un premier élément qui peut permettre de qualifier d'identitaire le parcours de l'histoire univer-

14 Peter Brown, interprète de La Cité de Dieu dans sa désormais classique biographie d'Augustin (La Vie de Saint Augustin, Paris, Seuil, 2001), affirme qu'Augustin « démolit » la culture païenne, non pas tant pourtant en tant que telle que parce qu' «idéalisant leur passé, les Romains font comme toute ciuitas terrena : ils prennent le transitoire pour absolu». (p. 405).

15 Voir Goulven Madec, "Le livre X du De ciuitate Dei : le sacrifice des Chrétiens ", Lettura del De ciuitate Dei di Agostino d'Ippona. Libri I-X, Lectio Augustini XV-XVI-XVII. Settimana Agostiniana Pavese (1999-2001), Roma, Institutum Patristicum Augustinianum (coll. "Studia Ephemeridis Augustinianum ", 86), p. 109-139.

16 Jean-Claude Guy, Unité et Structure logique de la Cité de Dieu de Saint Augustin, Paris, Études Augustiniennes, 1961. 
selle tel qu'Augustin le conduit des livres XI à XXII. Un second élément qui pourrait autoriser ce qualificatif est le fait qu'à la recherche, dans l'histoire, des traces d'une Cité de Dieu, Augustin qui ne les trouvait, jusqu'à la constitution d'un peuple avec Abraham, que dans le symbolisme (livre XV), les trouve désormais dans la mise à part d'une communauté préparée avec les Patriarches et instituée avec Moïse (c'est le livre XVI). Comme pour mieux souligner cette constitution identitaire d'une Cité de Dieu, Augustin lui oppose la concomitance de cités terrestres : les empires de Sicyonie, d'Égypte et d'Assyrie. Mais, selon lui, cette mise à part d'un Peuple de Dieu, sous la figure du peuple d'Israël, n'avait précisément comme fonction que de préfigurer l'avènement de l'Église. C'est le sens des prophéties qu'on peut trouver dans les livres des Prophètes mais aussi plus largement dans les textes poétiques et sapientiaux de la Bible (c'est le livre XVI). Cette inspiration divine des prophètes est d'ailleurs le signe d'une mise à part de la Cité de Dieu sous la figure d'Israël car Dieu n'a pas inspiré de la même façon les peuples grec et romain ni dans leur législation, ni dans leurs cultes restés polythéistes ni dans leur philosophie qui, aussi haute fût-elle, est toujours restée inférieure aux prophéties bibliques (c'est le livre XVIII). Enfin, un troisième argument qui pourrait permettre de qualifier ce parcours historique des livres XI à XXII d'identitaire est le fait qu'Augustin le fait triompher dans l'avènement de l'Église le jour de la Pentecôte (c'est le livre XVIII).

Au terme de ce premier temps de l'examen, on pourrait donc dire que $\mathrm{La}$ Cité de Dieu de Saint Augustin constitue, en effet, un essai d'histoire identitaire en réponse à un trouble politique puisque le point de départ de l'œuvre est bien l'immense trouble politique causé par le Sac de Rome le 24 août 410, objet du livre I, que les vingt et un autres livres ne semblent qu'une réponse à ce trouble et que cette réponse passe par une déconstruction de l'identité du paganisme au profit d'une construction identitaire chrétienne permettant de lire l'histoire, sous la seule autorité des Écritures chrétiennes, comme la mise à part d'une communauté triomphant dans l'Église.

Mais, comme nous l'avons rappelé dès l'introduction, s'agissant, avec $\mathrm{La}$ Cité de Dieu, d'une grande œuvre, nous devons être prudents et il est probable que l'œuvre ne peut se résumer, comme à une formule la définissant, à une tentative d'histoire identitaire en réponse à un trouble politique.

Bien sûr, La Cité de Dieu reste ancrée dans l'événement du Sac de Rome et il reste vrai que celui-ci a été un trouble politique comme nous l'avons dit tout à l'heure. Mais il faut tout de suite souligner qu'il n'a pas été un grand trouble pour Augustin déjà évêque d'Hippone. Un article de Jean Doignon publié dans la Revue des Études Augustiniennes ${ }^{17}$ oppose les réactions de Jérôme

17 Jean Doignon, "Oracles, prophéties, “on-dit” sur la chute de Rome (394-410). Les réactions de Jérôme et d'Augustin ", Revue des Études Augustiniennes, n 36, 1990, p. 120-146. 
et d'Augustin à l'annonce du Sac de Rome : à la panique de Jérôme, s'oppose la très grande relativisation de l'événement par Augustin. On peut, bien sûr, expliquer ces différences de réaction par des raisons psychologiques, mais, plus sérieusement, le clivage établi par Hervé Inglebert entre les théologies eusébiennes du pouvoir et les théologies non-eusébiennes voire anti-eusébiennes explique bien des choses ${ }^{18}$. En effet, les théologies eusébiennes du pouvoir voient, à la suite d'Eusèbe de Césarée, dans l'avènement d'un Empire chrétien à Rome, un événement décisif dans la réalisation du projet de Dieu dans l'histoire humaine et, dans la figure de l'empereur chrétien, un intermédiaire tout à fait particulier. On comprend que, dans cette perspective, Jérôme crie à la catastrophe lors du Sac de Rome. Au contraire, pour Augustin et La Cité de Dieu qu'Inglebert met en tête des réactions anti-eusébiennes ${ }^{19}$, la conversion de l'empereur, de l'Empire et de la ville de Rome n'ayant pas de signification particulière dans la théologie de l'histoire, le Sac de Rome perd de sa gravité. De la même façon, l'édition récente des cinq Sermons d'Augustin sur la Chute de Rome dans la Nouvelle Bibliothèque Augustinienne ${ }^{20}$ souligne le souci d'Augustin de relativiser l'événement en le replaçant dans une histoire non pas simplement plus longue mais plus haute, pourrait-on dire.

Si le Sac de Rome de 410 a été un trouble politique, c'est donc plus pour les destinataires de La Cité de Dieu que dans la pensée de son auteur. Que l'histoire prétendument identitaire parcourue dans les livres XI à XXII de La Cité de Dieu soit une "réponse " à ce trouble politique, cela aussi mérite d'être vérifié, notamment pour ce qui est le plus original dans ce parcours de l'histoire : sa relecture selon les deux concepts de "Cité terrestre » et de "Cité de Dieu ». Ces deux concepts, en effet, ne sont pas à proprement parler de véritables inventions d'Augustin et l'on a pu leur trouver de multiples sources possibles ${ }^{21}$; en revanche, leur application à la lecture de l'histoire est bel et bien une invention exclusive d'Augustin ${ }^{22}$. Or, depuis longtemps, André Lauras et Henri Rondet

18 Voir Hervé Inglebert, Les Romains chrétiens face à l'histoire de Rome : Histoire, christianisme et romanités en Occident dans l'Antiquité tardive, Paris, Études Augustiniennes, coll. "Série Antiquité ", 1996.

19 Voir Hervé Inglebert, op. cit., p. 395.

20 Voir Saint Augustin, Sermons sur la chute de Rome, éd. tr. fr. Jean-Claude Fredouille, Paris Turnhout, Institut d'Études augustiniennes - Brepols, coll. « Nouvelle Bibliothèque augustinienne », 8, 2004.

21 Voir J. Van Oort, Jerusalem and Babylon. A Study into Augustine's City of God and the Sources of His Doctrine of the Two Cities, Leyde, Brill, 1991.

22 Voir G. P. O'Daly, Augustine's City of God, op. cit., p. 62 : « La somme totale des influences possibles n'égale pas le champ sémantique du thème des deux cités et des thèmes connexes tels que nous les trouvons dans $\mathrm{La}$ Cité de Dieu. La synthèse d'Augustin va au-delà des idées et des textes qui ont pu l'influencer. Semble tout particulièrement originale l'application du motif des deux cités à un récit du cours de l'histoire (the sum total of possible influences does not equate with the scope of the theme of the two cities, and related themes, as we find them in the City of God. Augustine's synthesis is more than the ideas and texts that may have informed it. In particular, the application of the two cities model to an account of the course of history seems novel)." 
ont montré que cette invention, progressive chez Augustin, trouve son origine dans des œuvres anciennes et même dans des formulations et des applications très proches de La Cité de Dieu : dans des catéchèses, notamment, antérieures au Sac de Rome ${ }^{23}$. Loin d'être une invention " en réponse au trouble politique du Sac de Rome ", le concept des deux cités comme clef de lecture de l'histoire est donc chez Augustin une intuition ancienne à partir de laquelle il a réagi à l'événement du Sac de Rome plutôt qu'une idée qu'il aurait trouvée alors pour répondre au trouble politique.

Après le terme de "trouble politique ", de "réponse ", il nous reste à récuser la pertinence du terme d'" histoire identitaire " pour rendre compte de l'entreprise de La Cité de Dieu. Cette histoire, telle qu'elle est parcourue dans les livres XI à XXII pourrait être qualifiée d' "identitaire ", disionsnous, dans la mesure où, fondée sur la seule Auctoritas des Écritures chrétiennes, elle raconte la mise à part d'une communauté (le peuple d'Israël puis l'Église) et trouve son achèvement dans le triomphe de cette Église. Mais c'est ici qu'il faut revenir sur ce qui fait qu'on qualifie une démarche d' « identitaire ». Car, que cet adjectif récent en français soit employé, comme dans l'expression "construction identitaire " venant de la psychologie de l'éducation, avec une connotation positive ou qu'il garde de son origine dans la pensée d'extrême-droite une connotation négative comme dans les expressions " repli identitaire " ou " tentation identitaire ", il renvoie toujours à l'appartenance même symbolique à une communauté humaine définie comme exclusive des autres. Or il faut dire ici que, loin de coïncider avec une quelconque communauté humaine à laquelle elle s'identifierait, la Cité de Dieu augustinienne est transcendante, premièrement, à l'État chrétien, deuxièmement à l'Église visible si bien, que loin d'être identitaire, l'histoire parcourue par Augustin dans les livres XI à XXII de La Cité de Dieu est une histoire transcendante à l'histoire humaine elle-même.

Si - premièrement - la Cité de Dieu était identifiable à l'État chrétien, si du moins la réalisation d'un État chrétien était une étape importante dans la réalisation de la Cité de Dieu, Augustin aurait dû lui consacrer, à sa place dans le déroulement des livres XI à XXII, un développement important. Or il n'y a pas une ligne au livre XVIII sur l'Empire chrétien qui n'a donc aux yeux d'Augustin aucune signification au plan où il situe sa Théologie de l'Histoire $^{24}$. C'est en fait à la fin du livre V qu'Augustin parle de Constantin et de

23 Voir André Lauras et Henri Rondet, "Le Thème des deux Cités dans l'œuvre de saint Augustin ", Études Augustiniennes, Paris, Aubier, 1953, p. 99-162.

24 Cette absence est parfaitement cohérente avec la périodisation utilisée par Augustin dans La Cité de Dieu pour découper l'histoire humaine en six âges (correspondant aux six jours de la création et aux six âges de l'homme) puisque, l'histoire depuis la venue du Christ jusqu'à la fin du monde ne formant qu'un seul âge, elle ne peut être qu'homogène et ne peut comporter d'événements particulièrement signifiants du point de vue théologique. Voir, sur la périodisa- 
Théodose et qu'il fait l'éloge non pas tant de ces deux figures concrètes que du prince chrétien idéal ${ }^{25}$, se démarquant par là des panégyriques d'empereurs tels que les historiens eusébiens se plaisaient à les écrire et comme YvesMarie Duval l'a montré dans un article consacré à ces derniers chapitres du livre $\mathrm{V}^{26}$. D'ailleurs, comme aime à le répéter Augustin, ce n'est pas l'Empereur qui fait d'un Empire un Empire chrétien pas plus que ce ne sont les murs qui font la Cité mais les mœurs c'est-à-dire la conversion spirituelle et profonde de chacun des citoyens. Au prix de quel contre-sens lourd de conséquences la chrétienté médiévale s'est-elle appuyée sur La Cité de Dieu traduite pour Charles V par Raoul de Praelles pour étayer la théorie de l'État chrétien! C'est tout le paradoxe de la réception de l'œuvre montré par Étienne Gilson dans un petit livre Les Métamorphoses de La Cité de Dieu ${ }^{27}$ et qui mène de la pensée d'Augustin à sa déformation en augustinisme politique comme l'a montré un autre petit livre précieux : Augustin et l'Augustinisme d'Henri-Irénée Marrou ${ }^{28}$. Transcendante par rapport à toute incarnation politique, fût-elle chrétienne, la Cité de Dieu ne saurait être qualifiée d'identitaire dans la pensée d'Augustin pas plus que l'histoire qui voit sa réalisation progresser vers son achèvement.

Pas plus qu'elle ne s’identifie à l'État chrétien, la Cité de Dieu ne s'identifie à l'Église visible. Bien sûr des formules latines du type "ciuitas Dei siue ecclesia» ou "ciuitas Dei id est ecclesia ", lues dans La Cité de Dieu ${ }^{29}$ pourraient faire croire à une adéquation et à une identification de la Cité de Dieu à l'Église visible. Mais si, au lieu de se focaliser sur ces expressions, on essaie d'interpréter vraiment l'œuvre dans sa totalité, comme R. A. Markus dans Saeculum (op. cit.), on comprendra que «[p]lus profondément la "dichotomie radicale" qui caractérise les deux Cités dans leur réalité eschatologique s'oppose à l'identification de l'une comme de l'autre avec une société empirique quelconque ${ }^{30}$ ». De son côté, Henri-Irénée Marrou rappelle, dans son essai Théologie de l'Histoire ${ }^{31}$ - si nourri de La Cité de Dieu qu'il en consti-

tion de l'histoire dans La Cité de Dieu et sa signification, R. A. Markus, Saeculum: History and Society in The Theology of Saint Augustine, Cambridge, University Press, 1970, p. 20.

25 Voir Saint Augustin, La Cité de Dieu, V, XXIV-XXVI, 1.

26 Voir Yves-Marie Duval, «L'éloge de Théodose dans la Cité de Dieu (V, 26, 1). Sa place, son sens et ses sources ", Recherches Augustiniennes, n 4, 1966, p. 135-179. Voir aussi J.-M. Salamito, "Constantin vu par Augustin : pour une relecture de Civ. 5, 25 ».

27 Voir Étienne Gilson, Les Métamorphoses de la Cité de Dieu, Paris, Vrin, 1952.

28 Voir Henri-Irénée Marrou, Saint Augustin et l'Augustinisme, "Maîtres spirituels ", Paris, Seuil, 1971.

29 Voir par exemple La Cité de Dieu, XVI, II, 3 : " fideliter certum tenentes non ea sine aliqua praefiguratione futurorum gesta atque conscripta neque nisi ad Christum et eius ecclesiam, quae ciuitas dei est, esse referenda".

30 Revue des Études Augustiniennes et patristiques, "Bulletin Augustinien pour 1970 ", sur R. A. Markus, Saeculum..., op. cit., n 17, 1971.

31 Voir Henri-Irénée Marrou, Théologie de l'histoire, Paris, Seuil, 1968 / rééd., Cerf, 2006. 
tue comme un brillant commentaire - que, si l'on a une saine théologie de l'Église, le nom "ecclesia " ne doit pas d'abord renvoyer aux frontières visibles de l'Église " telle qu'elle est maintenant " mais plutôt à cette communauté spirituelle des saints et des élus qui sont appelés à vivre éternellement dans la vision de Dieu : l'Église « telle qu'elle sera alors » (c'est-à-dire après le Jugement dernier) constituée des anges, de tous les morts qui ont attendu le Messie avant sa venue, l'ont reconnu après et, plus largement encore, de tous ceux qui ont laissé leur raison reconnaître le Dieu unique en laissant leur poids, leur amour - c'est-à-dire la polarité de leur être profond - se diriger vers son lieu propre. Car c'est plus en ce sens ontologique que moral qu'il faut comprendre la célèbre définition des deux cités à la fin du livre XIV, définition qui, à elle seule, interdit de prendre la Cité de Dieu au sens identitaire de la communauté visible des seuls baptisés :

Deux amours ont fait deux cités : l'amour de soi jusqu'au mépris de Dieu a fait la cité terrestre ; l'amour de Dieu jusqu'au mépris de soi a fait la Cité céleste ${ }^{32}$.

D’ailleurs dans l'œuvre éponyme, la Cité de Dieu ne trouve sa réalisation, passant du statut de signum à celui de res, que par une opération de séparation par rapport à l'Église empirique mélangée, opération située lors du Jugement Dernier qui, seule, révélera la véritable Cité de Dieu définitive : l'Église eschatologique des hommes et des anges qui vivront éternellement dans la vision béatifique de Dieu.

La Cité de Dieu vers laquelle s'achemine l'Histoire ne s'identifiant ni à l'État chrétien ni à l'Église visible, le regard qu'Augustin jette sur l'Histoire loin d'être identitaire, est un essai d'histoire transcendante par rapport aux aléas de l'histoire humaine, parmi lesquels se trouve l'insignifiant Sac de Rome du 24 août 410. Il est clair que l'intégration à cette histoire du monde angélique et le fait de faire déboucher cette Histoire sur une Éternité sont des éléments qui permettent à Augustin de s'élever à une vision très haute de l'Histoire, à un point de vue surplombant sur le temps, un peu comme s'il se situait au point de vue de Dieu. On pourra trouver terriblement orgueilleuse cette posture et terriblement autocentrée cette lecture de l'Histoire sur la seule autorité des Écritures chrétiennes. Chacun jugera selon ses options personnelles.

Mais sur le terrain qui nous intéresse dans le cadre de l'université ouverte à l'universalité des savoirs, c'est-à-dire non pas tant la théologie catholique que l'histoire des idées, on ne pourra pas ne pas être admiratif devant l'effort de pensée incroyable dont a fait preuve Augustin dans La Cité de Dieu. Car le parcours de l'Histoire auquel il aboutit dans les livres XI à XXII de La Cité de Dieu est tout sauf identitaire. Il le serait si Augustin se situait au même

32 Saint Augustin, La Cité de Dieu, XIV, XXVIII : « Fecerunt itaque ciuitates duas amores duo, terrenam scilicet amor sui usque ad contemptum dei, caelestem uero amor dei usque ad contemptum sui". 
niveau que les Païens pour leur disputer l'interprétation du Sac de Rome et le récupérer dans une histoire où il serve les vues de la communauté chrétienne des années 410 ; on pourrait alors parler de conflit de mémoires. Mais, au contraire, ce à quoi l'on assiste dans ces livres, c'est, à l'occasion d'un trouble politique, l'éclosion du concept de Cité de Dieu, transcendante à toute cité en marche même chrétienne dans une histoire transcendante à toute histoire. Et cet effort pour hisser l'histoire - si l'on peut dire - à une hauteur de vue inégalée jusque-là a supposé un tel travail d'invention de concepts comme celui d'éternité ${ }^{33}$ ou de transformation de concepts déjà existants comme ceux de Providence, de cité, de Paix ou d'Autorité que nous pouvons parler d'invention d'une nouvelle mémoire non pas mise sur le même plan que celle des Païens mais pensée comme supérieure ${ }^{34}$. Et Henri-Irénée Marrou dans Théologie de l'Histoire a vu, dans cet effort de pensée, comme un sommet inégalé puisque les philosophies de l'histoire ultérieures, qu'elles soient hégéliennes, marxistes ou qu'elles se réduisent à l'idéologie du progrès, ne sont, selon lui, que de piètres avatars de cette Théologie augustinienne de l'Histoire en ce qu'elles rabaissent au niveau terrestre le but ultime de l'Histoire qu'est, selon Augustin, la suprême béatitude dans la vision éternelle de Dieu.

Philippe Cournault Centre Paul-Albert Février (UMR 7297) Aix-en-Provence p.cournault@cpe.leschartreux.net

\begin{abstract}
Résumé
À première vue, La Cité de Dieu de Saint Augustin semble bien être une histoire identitaire en réponse à un trouble politique puisque, en réponse au Sac de Rome de 410, elle déconstruit le système politico-religieux du paganisme pour lui substituer l'Auctoritas des Écritures chrétiennes à partir desquelles elle relit toute l'histoire universelle. Mais, en réalité, tout cet effort n'est pas identitaire car la Cité de Dieu, aboutissement de cette histoire, transcende la communauté de l'Église et cette histoire transcende toute histoire particulière.
\end{abstract}

\title{
Mots-clés
}

Cité de Dieu, trouble politique, histoire identitaire, Histoire transcendante, auctoritas.

33 Du moins en son sens augustinien de temps dégagé de toute successivité contrairement à la " fausse " éternité des Platoniciens réduite, selon Augustin, à n'être qu'une alternance cyclique.

34 Voir Philippe Cournault, La Cité de Dieu de Saint Augustin : une auvre de mémoire, thèse P. Cambronne (éd.), université Michel-de-Montaigne-Bordeaux 3, 2011. 


\section{Abstract}

At first glance, The City of God by Saint Augustin seems to be a work focused on "identity" history resulting from political unrest since, in response to the Sack of Rome in 410, it deconstructs the political and religious system on which paganism is based to substitute it with the Auctoritas of the Christian Scriptures which it then uses to link together the whole of universal history. But, in fact, this is not the case because The City of God where this history leads to, transcends the community of the Church and this story itself transcends every particular history.

\section{Keywords}

City of God, political unrest, identity history, transcendant History, auctoritas. 